\title{
Heat Transfer and Flow Characteristics in a Tube with Small Tube Inserts in a Pulsating Flow
}

\author{
Huan-ling Liu ${ }^{1,2}$, Ping liu ${ }^{2}$, David Nobes ${ }^{3}$ \\ ${ }^{1}$ Xidian-Ningbo Information Technology Institute Xidian University \\ Xi'an, 710071, China \\ hlliu@xidian.edu.cn \\ ${ }^{2}$ School of Electromechanical Engineering, Xidian University \\ Xi'an, 710071, China \\ liuping@stu.xidian.edu.cn \\ ${ }^{3}$ Department of Mechanical Engineering, University of Alberta \\ Edmonton, Alberta, Canada, T6G 1H9 \\ david.nobes@ualberta.ca
}

\begin{abstract}
The heat transfer and flow of a water- $\mathrm{TiO}_{2}$ nano-fluid in horizontal tube fitted with mixing inserts was studied by numerical simulation. The effects of sinusoidal amplitude and period of the fluid velocity were investigated. A cyclical variation in the friction coefficient, $f$, and the Nusselt number, $\mathrm{Nu}$, that is proportional to the amplitude was observed. The larger the amplitude, the greater the heat transfer and drag coefficient. In general, the overall evaluation factor (PEC) of cases with higher oscillating amplitudes was larger. The maximum was achieved for the case with highest oscillating amplitude but lowest period. The simulation also showed that the inserts result in a local change in the flow direction from parallel-to-perpendicular to the wall, which decrease the angle between velocity vector and temperature gradient and leads to heat transfer enhancement.
\end{abstract}

Keywords: Numerical Simualtion, Pulsating Flow, Water-Tio 2 Nano-Fluid, Heat Transfer, Mixing Inserts.

\section{Introduction}

With the miniaturization of electronic equipment and continuing development of integrated circuit often comes a significant increase in the heat flux generated by the system [1]. Continuing efforts have been underway to promote the heat transfer coefficient and keep the performance of such equipment at an optimal level [2-3]. Enhancing the heat transfer performance is, nevertheless, an active research area in the field.

Nano-fluids [4] have been widely used as the working fluid since 1995. The choice primarily follows their high efficiency in heat conduction, stability and low pressure drop properties [5]. The thermal conductivity, viscosity and other thermal properties of nano-fluids has been the subject for a growing number of research studies [6-9]. For example, the thermal conductivity and viscosity of $\mathrm{TiO}_{2}$ of $3 \%$ concentration in the temperature range of $13{ }^{\circ} \mathrm{C}$ and $55{ }^{\circ} \mathrm{C}$ has been studied [10]. It indicated that the enhancement of thermal conductivity was observed to be $7.4 \%$ over the base fluid at $13 \mathrm{C}$ and the increase in the nano-fluid viscosity is larger than the enhancement in the thermal conductivity.

The characteristics of pulsatile flow in different channels and the use of various types of flow spoilers have been widely studied by theoretical analysis, experimental and numerical techniques [11-17]. It has been shown that, for a uniform heat flux, the heat transfer in pulsating flow (frequency $=1-4 \mathrm{~Hz}$ ) in the laminar regime $(R e=1366)$ can be enhanced by $30 \%$ [11]. Mackley et al. [12] reported that the pulsating flow had no effect on the heat transfer characteristics of plain tubes. It was shown however, that twist inserts enhanced the heat transfer in compact structures [13]. Ferroni [14] conducted experiments in circular tube equipped with physically separated, multiple, short-length twisted tapes and reported that pressure drop with multiple short-length twisted-tapes were at least 50\% lower than full-length twisted-tapes. Tu et al. [15] numerically studied the heat transfer and friction characteristics in a tube with small tube inserts and reported the maximal Nusselt number was enhanced by 3.4-10.3 times as that of the smooth tube by the addition of three pipe inserts. 
It can be observed that the literature has much more coverage in the topic of laminar pulsating flow than turbulent flow. In turbulent flows, small tube inserts can reduce the boundary layer to enhance heat transfer [18]. By using nanofluids, however, higher levels of heat transfer enhancement can be achieved. In this study, the heat transfer enhancement and reduction in pressure drop as a result of $\mathrm{TiO}_{2}$ nano-fluids with a concentration of $1 \%$ in tubes fitted with new inserts are numerically studied under influence of a pulsatile flow with a sine velocity function.

\section{Problem Description}

The flow domain under investigation that includes the straight tube installed with the small inserts is shown in Fig. 1. Six small tube inserts which are composed of a main tube and three branches can be seen in the figure. The six inserts are separated to decrease the pressure drop. The inserts are mainly used to introduce nano-fluids to the wall. The inner diameter of the pipe $D$ is $0.016 \mathrm{~m}$, respectively. The total length of the tube is $1.5 \mathrm{~m}$. The diameter of the branch tube $d$ is $0.002 \mathrm{~m}$, the length of the insert $l$ is $0.015 \mathrm{~m}$. The distance between two inserts is $0.035 \mathrm{~m}$ and the angle between the branch tube and the main tube $\delta$ is $67^{\circ}$.

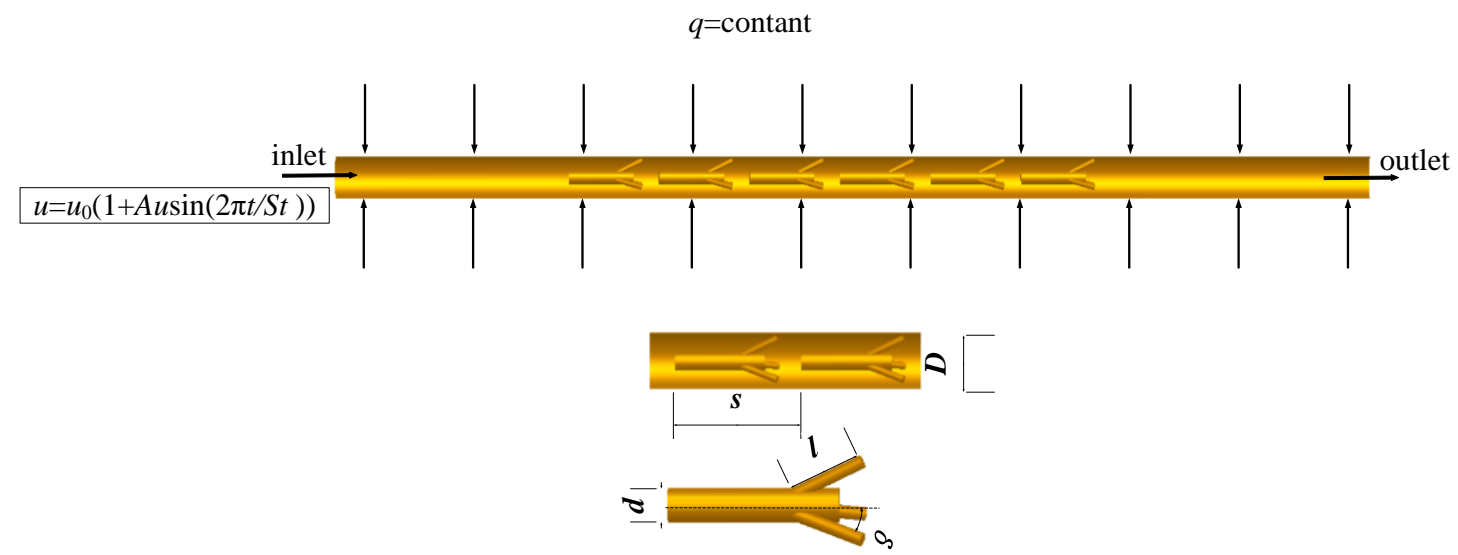

Fig. 1: Computational domain and relevant geometrical parameters.

\subsection{Governing Equations and Boundary Conditions}

For an incompressible flow, using the generated notations $x_{1} \equiv x, x_{2} \equiv y, x_{3} \equiv z, u_{1} \equiv u, u_{2} \equiv v$ and $u_{3} \equiv w$, the continuity, momentum and energy equations for a fluid with constant thermal properties can be written as:

$$
\begin{gathered}
\partial\left(\rho_{f} u_{i}\right) / \partial x_{i}=0 \\
\partial\left(\rho_{f} u_{i}\right) / \partial t+\partial\left(\rho_{f} u_{i} u_{j}\right) / \partial x_{i}=-\partial p / \partial x_{i}+\partial\left\{\mu_{f}\left(\partial u_{j} / \partial x_{i}+\partial u_{i} / \partial x_{j}\right)\right\} / \partial x_{i}, j=1,2,3 \\
\partial\left(\rho_{f} T\right) / \partial t+\partial\left(\rho_{f} u_{i} T\right) / \partial x_{i}=-\partial\left(\lambda_{f} \partial T / \partial x_{i}\right) / \partial x_{i}
\end{gathered}
$$

where $p$ is the pressure, $T$ the temperature, $\lambda_{f}$ the fluid heat conductivity, $\rho_{f}$ the density, $u$ the velocity of $x$ direction, $v$ the velocity of $y$ direction, $w$ the velocity of $\mathrm{z}$ direction, $\mu_{f}$ the dynamic viscosity and $t$ time. Since the governing equations are not for a closed control volume, boundary conditions need to be established for all boundaries in the computational domain. The inlet boundary conditions can be described by:

$$
u=u_{0}\left(1+A u \sin \left(\frac{2 \pi}{S t} t\right)\right), \quad u=w=0, \quad T=T_{i n}
$$


where $u_{0}$ is the mean velocity at the inlet $(0.55 \mathrm{~m} / \mathrm{s}), A u$ is the non-dimensional oscillating amplitude, $T u$ is the oscillating period, $S t$ is the Strouhal number $\left(S t=\frac{D}{u T u}\right.$ ) and $T_{\text {in }}$ the inlet temperature. The outflow condition is used at the outlet. A wall heat flux boundary condition of $q=6731 \mathrm{~W} / \mathrm{m}^{2}$ was used to continuously provide heat to the system. The wall of inserts where insulated and the no slip velocity boundary condition was use for all internal surface.

\subsection{Physical Properties of Nanofluids}

The density and specific heat relationships of a nano-fluid have been determined by Azmi [9] and can be expressed as:

$$
\begin{gathered}
\rho_{n f}=\phi \rho_{p}+(1-\phi) \rho_{w} \\
c_{n f}=\frac{(1-\phi)(\rho C)_{w}+\phi(\rho C)_{p}}{(1-\phi) \rho_{w}+\phi \rho_{p}}
\end{gathered}
$$

where $\rho, C$ and $\phi$ is the density, specific heat capacity and volume fraction, respectively. Subscript $n f, p, w$ refers to nanofluid, particle and water.

The viscous coefficient and thermal conductivity are to be evaluated for a concentration of the nano-fluid within the range of $0.5 \%-4.0 \%$. A set of regression equations given in Eq. (7) and Eq. (8) below was forwarded by Sharma et al. [10] based on results from experiments that used water as the base fluid.

$$
\begin{gathered}
\mu_{f}=\frac{\mu_{n f}}{\mu_{w}}=\left(1+\frac{\phi}{100}\right)^{11.3}\left(1+\frac{T_{n f}}{70}\right)^{-0.038}\left(1+\frac{d_{p}}{170}\right)^{-0.061} \\
\lambda_{f}=\frac{\lambda_{n f}}{\lambda_{w}}=0.8938\left(1+\frac{\phi}{100}\right)^{1.37}=\left(1+\frac{T_{n f}}{70}\right)^{0.27777}\left(1+\frac{d_{p}}{150}\right)^{-0.0336}\left(\frac{\alpha_{p}}{\alpha_{w}}\right)^{0.01737}
\end{gathered}
$$

Where $\mathrm{d}$ is the partical diameter, $\alpha$ thermal diffusivity. It should be noted here that these equations apply only for cases where the concentration and the fluid temperature values are less than $4 \%$ and $70{ }^{\circ} \mathrm{C}$ respectively and the particle size cannot be larger than $170 \mathrm{~nm}$. The physical properties of $\mathrm{TiO}_{2}$ and the base fluid (water) are given in Table 1.

Table 1: Physical properties of $\mathrm{TiO}_{2}$, Water and $\mathrm{TiO}_{2}$-water at $30{ }^{\circ} \mathrm{C}$.

\begin{tabular}{|l|l|l|c|}
\hline & $\mathrm{TiO}_{2}$ & Water & $\mathrm{TiO}_{2}$-water \\
\hline$k, \mathrm{~W} / \mathrm{m} . \mathrm{K}$ & 8.4 & 0.618 & 0.9742 \\
\hline$\rho, \mathrm{Kg} / \mathrm{m}^{3}$ & 4175 & 995.7 & 1047.4 \\
\hline$C_{\mathrm{p}}, \mathrm{J} / \mathrm{kg} . \mathrm{K}$ & 692 & 4178.4 & 4138.86 \\
\hline
\end{tabular}

\subsection{Definition of Parameters}

For this study the Reynolds number of the flow, $R e$, was defined as:

$$
R e=\frac{\rho \mu D_{e}}{\mu} \quad D_{e}=\frac{4 A_{c}}{P}
$$

where $D_{e}$ and $A_{c}$ are the hydraulic diameter and the area of the channel cross section, respectively and $P$ is the wetted the perimeter (circumference).

The Nusselt number, $N u$ was defined as in [19] such that: 


$$
\begin{array}{r}
N u_{x, t}=\frac{h_{x, t} D e}{\lambda} \\
N u_{t}=\frac{1}{L} \int_{0}^{L} N u_{x, t} d x \\
N u=\frac{1}{\tau} \int_{0}^{\tau} N u_{t} d t
\end{array}
$$

where $\tau$ is the period of the oscillation, $N u$ is the Local Nusselt number, $N u_{x, t}$ is the spatial and $N u$ is the time-spatialaveraged Nusselt

The heat transfer coefficient was defined as:

$$
h_{x, t}=\frac{Q_{i}}{A \Delta T_{(x, t)}}
$$

where $A$ is the area of interface between the fluid and the wall, $Q$ is the local heat transfer rate, $\Delta T(x, t)$ is the gradient in average wall temperature $T_{w}(x, t)$ and the fluid bulk temperature $T_{f}(x, t)$.

According to the definition of the flow resistance characteristics [20], the global and local friction coefficient can be expressed as follows

$$
\begin{gathered}
f=\frac{p_{\text {in }}-p_{\text {out }}}{\frac{1}{2} \rho u^{2}} \frac{D_{e}}{L} \\
f_{i}=\frac{p_{i}{ }^{\prime}-p_{i}{ }^{\prime \prime}}{1 / 2 \rho u^{2}} \frac{D_{e}}{L_{i}}
\end{gathered}
$$

where $p_{i n}, p_{\text {out }}, p_{i}^{\prime}$ and $p_{i}^{\prime \prime}$ are the total mass-weighted average pressures at global sections - inlet, and outlet, - and local sections.

To evaluate the overall system, Webb [21] developed an overall performance evaluation criterion (PEC) defined as:

$$
P E C=\frac{N u N u_{0}^{-1}}{\left(f f_{0}^{-1}\right)^{1 / 3}}
$$

where $N u_{0}$ and $f_{0}$ is the Nusselt number and friction coefficient of plain tube.

To undertake the simulations, a commercial software for computational fluid dynamics (CFD) (Fluent 14.5, ANSYS, Inc) was used in this study. A hexahedral structured grid for the outer tube, and the o type grid for the inner tube were generated. An encrypted grid was used for the boundary layer. The finite-volume method was used to solve the governing equations given in Eq. (1) - (3) before applying the SIMPLEC algorithm to solve the resulting differential equations to obtain the velocity, pressure and temperature fields in the control volume. The standard pressure and second-order upwind discretization schemes for momentum and energy equations were employed in the numerical model. A convergence criteria of $10^{-5}$ was used for the continuity, momentum, and energy equations. To study the heat transfer and flow characteristics, the numerical simulation was conducted for $A u=0.75$ and $S t=0.0097$.

\subsection{Grid and Time Step Independent Tests and Validations}

A grid independence analysis was conducted on six different grid systems with 44,849, 87759, 167,757, 378480, 607867, and 1014467 grids to validate the precision of the numerical solution. The test results of grid independence are 
shown in Figure 2. It can be seen that both of the simulated $N u$ number and $f$ number achieve a constant value for grid numbers greater or equal to 607,867 . This number of grids was, therefore, found to be sufficient for grid-independent solutions.

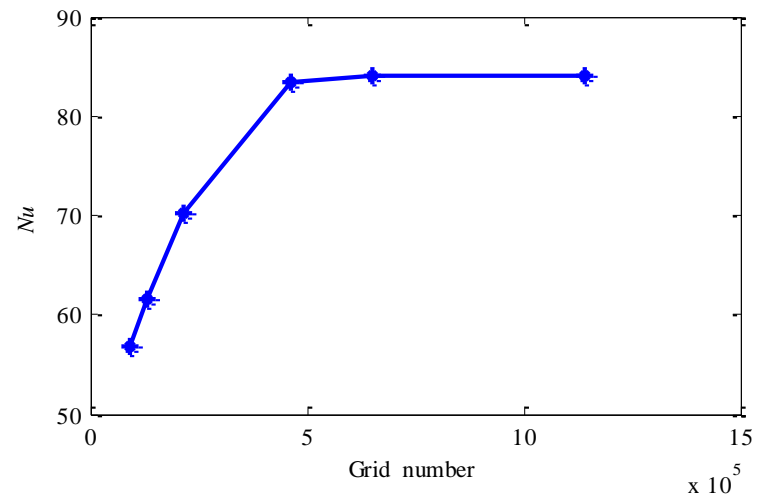

(a) $\mathrm{Nu}$ versus grid number

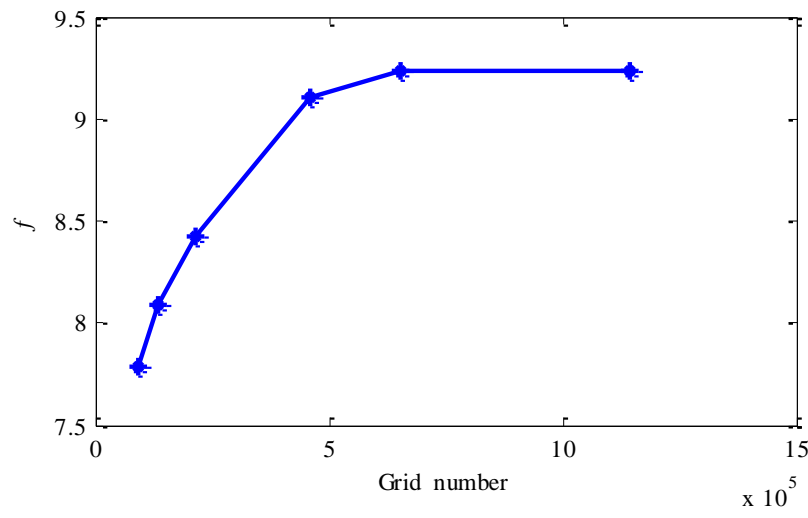

(b) $f$ versus grid number

Fig. 2: Simulation tests comparing (a) $N u$ and (b) $f$ for grid-independent solutions.

\section{Results and Discussions}

\subsection{Heat Transfer and Flow Performance}

The velocity distributions at 1/4 and 3/4 cycles are shown in Fig. 3. As shown in Fig. 3(a), the velocity direction changes dramatically from streamline and parallel to the wall due to the branch tube. However, the velocity of the branch tube is nearly zero. It can be seen that the velocity at $1 / 4$ period is higher than at $3 / 4$ period of larger velocity and developing boundary layer are therefore observed. The flow has a higher velocity at the inlet to the branch tubes and close to the outlet. This is due to the sudden change of direction the flow encounters as it reaches the branch pipe connection. As the fluid flows through the charge connected with the tubular spoiler, both the magnitude and direction of the velocity change rapidly. The need to overcome the local resistance at $3 / 4$ cycle results in a low velocity in the main channel, which in turn leads to a near-zero velocity in the branches.

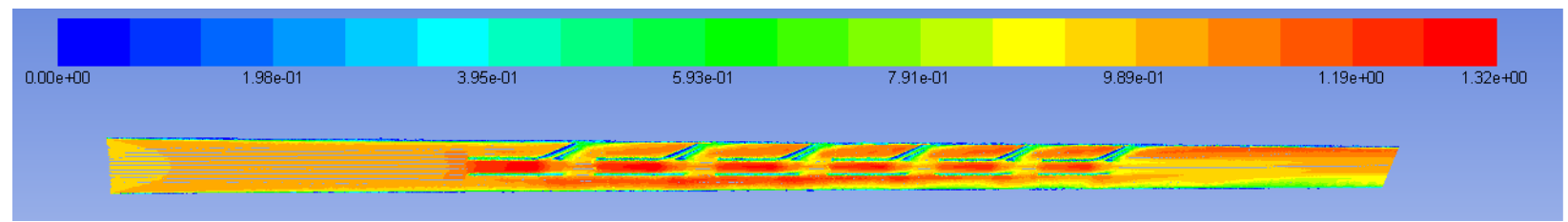

(a) $1 / 4$ period for

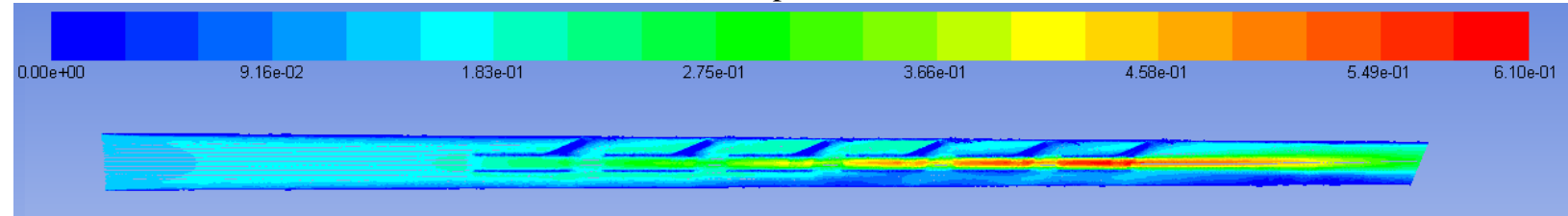

(b) $3 / 4$ period for

Fig. 3: The fluid velocity distribution for $1 / 4$ and $3 / 4$ period.

The variations in $N u$ and $f$ as a function of $\Phi$ in one cycle are shown in Fig. 4. It can be seen that the $N u$ number and $f$ fluctuate around the corresponding steady-state values, and both amplitudes of $N u$ and $f$ have higher upward fluctuations than downward fluctuation. The average $N u$ is greater than that of the steady state value. The fluctuation amplitudes of $N u$ and $f$ also increase as the ripple amplitude increases. This is because the velocity at which the fluid flows through the branch pipe to the wall results in a higher velocity gradient. At the same time, the shear stress between the fluid and the wall increases leading to higher resistance. By numerical calculation, the average $N u$ number in a cycle increased by $22 \%$. 
The friction factor also increased by $13.8 \%$. Furthermore, by employing Eq. (16), the $P E C$ number equals to 1.17 is obtained, which would be used in engineering applications for heat transfer enhancement since $P E C>1$.

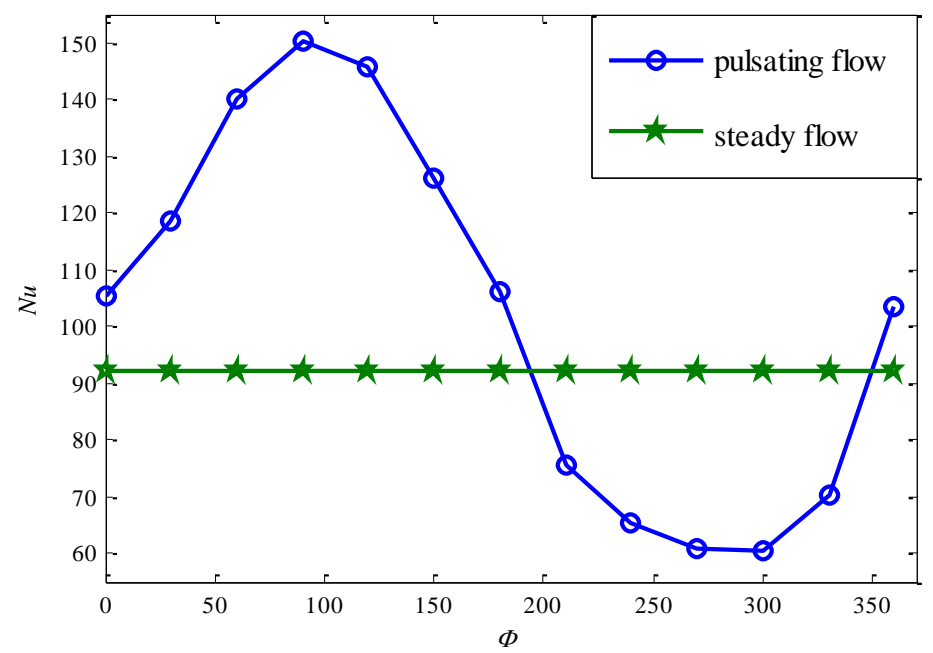

(a) $N u$ versus $\Phi$

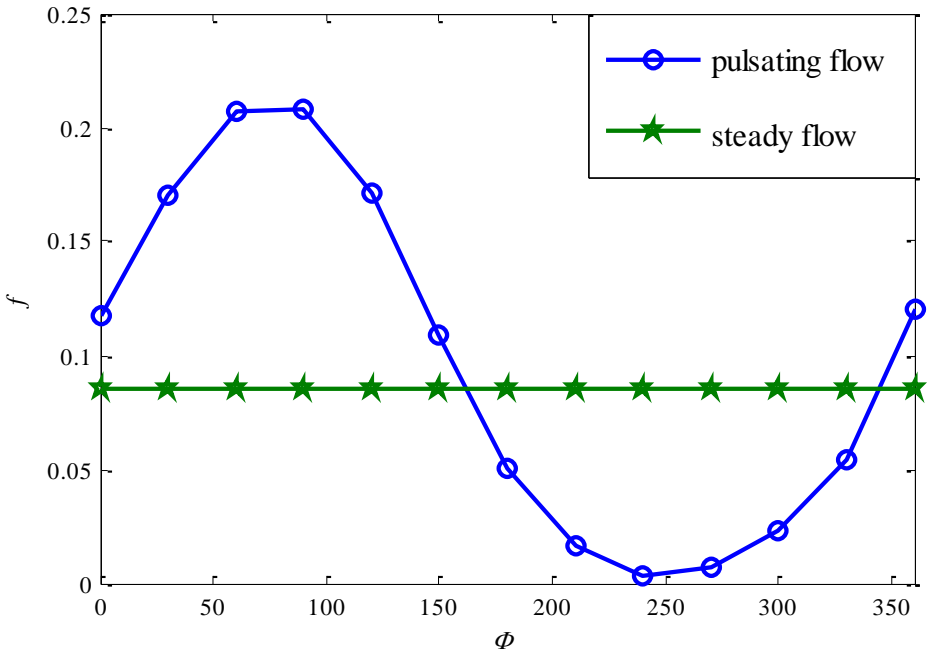

(b) $f$ versus $\Phi$

Fig. 4: The effects of amplitude and period on heat transfer in a period.

\section{Conclusion}

The flow and heat transfer characteristics of turbulent flow in a tube fitted with small pipe inserts with sinusoidal inlet velocity were studied by numerical simulation. The main conclusions drawn are:

(1) The velocity direction changes dramatically from streamline and parallel to the wall due to the branch tube.

(2) For pulsating flow, the average friction coefficient and the average Nusselt number are proportional to the amplitude in one period.

(3) The average Nusselt number in a cycle increased by $22 \%$ with resistance punishment $13.8 \%$.

(4) The overall heat transfer evaluation factors of $P E C$ can reached 1.17 with $A u=0.75$ and $S t=0.0097$

\section{Acknowledgements}

This work is supported by the Ningbo Natural Science Foundation for Xidian-Ningbo Information Technology Institute (No.2017A610031) and the China Scholarship Council (No.201706965047) and the 111 Project (No.B14042).

\section{References}

[1] I. Ferain, C. A. Colinge and J. P. Colinge, "Multigate transistors as the future of classical metal-oxidesemiconductor field-effect transistors," Nature, vol. 479, no. 17, pp. 310-316, 2011.

[2] K. Kouloulias, A. Sergis and Y. Hardalupas, "Sedimentation in nanofluids during a natural convection experiment," Int. J. Heat Mass Tran., vol. 101, pp. 1193-1203, 2016.

[3] S. Choi, "Enhancing thermal conductivity of fluids with nanoparticles developments and applications of on Newtonian flows," Applied Physics A, vol. 66, pp. 99-105, 1995.

[4] P. Keblinski1, J. A. Eastman and D. G. Cahill, "Nanofluids for thermal transport," Materials Today, vol. 8, no. 6, pp. 36-44, 2005.

[5] K. Khanafer, K. Vafai and M. Lightstone, "Buoyancy driven heat transfer enhancement in a two-dimensional enclosure utilizing nanofluids," Int. J. Heat Mass trans, vol. 46, no. 9, pp. 3639-3653, 2003

[6] M. Liu , M. C. Lin and C. Wang, Enhancements of thermal conductivities with $\mathrm{Cu}, \mathrm{CuO}$, and carbon nanotube nanofluids and application of MWNT/water nanofluid on a water chiller system," Nanoscale Res. Lett., vol.6, pp. 297-310, 2011. 
[7] J. A. Eastman, S. U. S. Choi, S. Li, W. Yu, and L. J. Thompson, "Anomalously increased effective thermal conductivities of ethylene glycol-based nanofluids containing copper nanoparticles," Appl. Phys. Lett., vol. 78, pp. 718-720, 2001.

[8] C. H Li, and G. P. Peterson, "Experimental investigation of temperature and volume fraction variations on the effective thermal conductivity of nanoparticle suspensions (nanofluids)," J. Appl. Phys., vol. 99, 2006.

[9] H. Masuda, A.Ebata and T. Kazunari, "Alteration of thermal conductivity and viscosity of liquid by dispersing ultra fine particles Netsu Bussei,” Netsu Bussei (Japan), vol. 4, no. 4, pp. 227-233,1993.

[10] A. Turgut, I. Tavman, M. Chirtoc, H. P. Schuchmann, C. Sauter and S. Tavman, "Thermal conductivity and viscosity measurements of water-based TiO2 nanofluids," Int. J. Thermophys, vol. 30, pp. 1213-1226, 2009.

[11] M. A. Habib, A. M. Attya and A. I. Eid, "Convective heat transfer characteristics of laminar pulsating pipe air flow," Heat Mass Tran., vol. 38, no. 3, pp. 221-232, 2002.

[12] M. R. Mackley, G. M. Tweddle and I.D. Wyatt, "Experimental heat transfer measurements for pulsatile flow in baffled tubes," Chem. Eng. Sci.,vol. 45, no. 5, pp. 1237-1242,1990.

[13] H. Bas and V. Ozceyhan, "Heat transfer enhancement in a tube with twisted type inserts placed separately from the tube wall," Exp. Therm. Fluid Sci., vol. 41, no. 9, pp. 51-58, 2012.

[14] P. Ferroni, R. E.Block, N. E. Todreas and A.E. Bergles, "Experimental evaluation of pressure drop in round tubes provided with physically separated, multiple, short-length twisted tapes," Exp. Therm. Fluid Sci., vol. 35, no. 7, pp.1357-1369, 2011.

[15] W. B. Tu, T. Yong, J. Y. Hu, Q. H. Wang and L. S. Lu, "Heat transfer and friction characteristic of laminar flow through a circular tube with small pipe inserts," Int J Therm. Sci., vol. 96, no. 10, pp. 94-101, 2015.

[16] W. H. Azmia, K. V. Sharmab, P. K. Sarma, R Mamata and G. Najafid, "Heat transfer and friction factor of water based $\mathrm{TiO}_{2}$ and $\mathrm{SiO}_{2}$ nanofluids under turbulent flow in a tube," Int. Com. in Heat and Mass Transf., vol. 59, no. 12, pp. 30-38, 2014.

[17] K. V. Sharma, P. K. Sarm, W. H. Azmi, R. Mamat and K. Kadirgama, "Correlations to predict friction and forced convection heat transfer coefcients of water based nanouids for turbulent flow in a tube," Int. J. Microscale Nanoscale Therm Fluid Trans. Phenom., vol. 3, no. 4, pp. 1-25, 2012.

[18] M. Siddique, A. R. A. Khaled, N. I. Abdulhafiz and A. Y. Boukhary, "Recent Advances in Heat Transfer Enhancements: A Review Report," Int. J. Chem. Eng., vol. 2010. DOI:10.1155/2010/106461

[19] F. Selimefendigil, H. F Oztop, "Control of Laminar Pulsating Flow and Heat Transfer in Backward-Facing Step by using a Square Obstacle," Journal of Heat Transfer, vol. 136, no. 8, pp.081701-081711, 2014.

[20] Y. Wang, Y. L. He, W. W. Yang and Z. D. Cheng, "Numerical analysis of flow resistance and heat transfer in a channel with delta winglets under laminar pulsating flow," Int. J. Heat and Mass Tran., vol. 82, pp. 51-65, 2015.

[21] R. L.Webb, "Performance evaluation criteria for use of enhanced heat transfer surfaces in heat exchanger design," Int. J. Heat Mass Tran., vol. 24, no. 4, pp. a715-726, 1981. 